\title{
Clinical insights gained by refining the 2016 WHO classification of diffuse gliomas with: EGFR amplification, TERT mutations, PTEN deletion and MGMT methylation
}

Cheila Brito ${ }^{1 \dagger}$, Ana Azevedo ${ }^{2,3 \dagger}$, Susana Esteves ${ }^{4}$, Ana Rita Marques ${ }^{1}$, Carmo Martins ${ }^{1}$, Ilda Costa ${ }^{2}$, Manuela Mafra ${ }^{5}$, José M. Bravo Marques², Lúcia Roque ${ }^{1+}$ and Marta Pojo ${ }^{1 *+}$ (D)

\begin{abstract}
Background: Significant advances in the molecular profiling of gliomas, led the 2016 World Health Organization (WHO) Classification to include, for the first-time, molecular biomarkers in glioma diagnosis: IDH mutations and 1p/ $19 \mathrm{q}$ codeletion. Here, we evaluated the effect of this new classification in the stratification of gliomas previously diagnosed according to 2007 WHO classification. Then, we also analyzed the impact of TERT promoter mutations, PTEN deletion, EGFR amplification and MGMT promoter methylation in diagnosis, prognosis and response to therapy in glioma molecular subgroup.
\end{abstract}

Methods: A cohort of 444 adult gliomas was analyzed and reclassified according to the 2016 WHO. Mutational analysis of IDH1 and TERT promoter mutations was performed by Sanger sequencing. Statistical analysis was done using SPSS Statistics 21.0.

Results: The reclassification of this cohort using 2016 WHO criteria led to a decrease of the number of oligodendrogliomas (from 82 to 49) and an increase of astrocytomas (from 49 to 98), while glioblastomas (GBM) remained the same $(n=256)$. GBM was the most common diagnosis $(57.7 \%)$, of which $55.2 \%$ were IDH-wildtype. $1 p / 19 q$ codeleted gliomas were the subgroup associated with longer median overall survival (198 months), while GBM IDH-wildtype had the worst outcome (10 months). Interestingly, PTEN deletion had poor prognostic value in astrocytomas IDH-wildtype $(p=0.015)$, while in GBM IDH-wildtype was associated with better overall survival $(p=$ $0.042)$ as well as MGMT promoter methylation $(p=0.009)$. EGFR amplification and TERT mutations had no impact in prognosis. Notably, EGFR amplification predicted a better response to radiotherapy $(p=0.011)$ and MGMT methylation to chemo-radiotherapy $(p=0.003)$.

Conclusion: In this study we observed that the 2016 WHO classification improved the accuracy of diagnosis and prognosis of diffuse gliomas, although the available biomarkers are not enough. Therefore, we suggest MGMT promoter methylation should be added to glioma classification. Moreover, we found two genetic/clinical correlations that must be evaluated to understand their impact in the clinical setting: i) how is PTEN deletion a favorable prognostic factor in GBM IDH wildtype and an unfavorable prognostic factor in astrocytoma IDH wildtype and ii) how EGFR amplification is an independent and strong factor of response to radiotherapy.

Keywords: EGFR, TERT, MGMT, PTEN, IDH, 1p/19q codeletion, 2016 WHO classification, Gliomas

\footnotetext{
* Correspondence: martapojo@gmail.com

${ }^{\dagger}$ Cheila Brito, Ana Azevedo, Lúcia Roque and Marta Pojo contributed equally to this work

${ }^{1}$ Unidade de Investigação em Patobiologia Molecular (UIPM) do Instituto Português de Oncologia de Lisboa Francisco Gentil E.P.E., Rua Prof. Lima Basto, 1099-023 Lisbon, Portugal

Full list of author information is available at the end of the article
}

(c) The Author(s). 2019 Open Access This article is distributed under the terms of the Creative Commons Attribution 4.0 International License (http://creativecommons.org/licenses/by/4.0/), which permits unrestricted use, distribution, and reproduction in any medium, provided you give appropriate credit to the original author(s) and the source, provide a link to the Creative Commons license, and indicate if changes were made. The Creative Commons Public Domain Dedication waiver (http://creativecommons.org/publicdomain/zero/1.0/) applies to the data made available in this article, unless otherwise stated. 


\section{Background}

Diffuse gliomas are one of the most common primary neoplasms of the central nervous system, accounting for approximately $81 \%$ of all malignant brain tumors, leading to a high rate of mortality and morbidity [1, 2]. These aggressive and heterogeneous tumors are generally associated with poor outcomes, due to their complexity and resistance to therapeutic approaches [3].

In the last years, improvements in molecular techniques have been important tools to update the knowledge about the genetic profile of gliomas. These progresses, led in 2016, the World Health Organization (WHO) classification of Central Nervous System Tumors to include Isocitrate dehydrogenase $(I D H)$ mutations and $1 \mathrm{p} / 19 \mathrm{q}$ codeletion as central biomarkers for the diagnosis of diffuse gliomas [4]. This new classification breaks the principle of diagnosis based exclusively on microscopy, allowing a more accurate determination of the patient's prognosis $[4,5]$. Nevertheless, this new classification has limitations to characterize these heterogeneous tumors. New biomarkers for diagnostic, prognostic and response to therapy are a major concern for the management of patients with gliomas [6]. In this context, different potential biomarkers for diffuse gliomas have been proposed, such as: TERT (telomerase reverse transcriptase) promoter mutations, amplification/mutations in $E G F R$ (epidermal growth factor receptor) gene, mutations/ deletions in PTEN (phosphatase and tensin homologue) and MGMT (O-6-methylguanine-DNA methyltransferase) promoter methylation.

TERT promoter mutations are present in a high percentage of gliomas (80-90\%), which makes it an interesting target gene to be studied [7]. This gene encodes the catalytic subunit of telomerase, an enzyme that maintains the length of telomeres during cell division [8]. In addition, TERT promoter mutations are associated with increased levels of telomerase activity allowing the indefinite proliferation of tumor cells [8-10]. The amplification of EGFR was identified in approximately $40-50 \%$ of all cases of glioblastoma (GBM), 2007 WHO grade IV, the most malignant of diffuse gliomas [11, 12]. This molecular alteration determines the over-activation of an important signaling pathway, phosphatidylinositol-3kinase - protein kinase B (PI3K-AKT), which regulates a wide range of cellular processes such as cell proliferation, migration, angiogenesis, differentiation and apoptosis [13].

PTEN deletion is present in approximately $30-40 \%$ of GBM $[14,15]$, however there is no unanimity regarding the prognostic value of this alteration in diffuse gliomas $[16,17]$, as well as, regarding TERT promoter mutations [18-20] and EGFR amplification [21-23].

$M G M T$ promoter methylation has been described as a predictive biomarker in GBM with benefit from chemotherapy based on temozolomide [24-26]. Moreover, this benefit is higher in patients with $I D H$-wildtype gliomas, particularly in old patients (aged $\geq 70$ years) $[3,27,28]$. MGMT promoter methylation is predominant in $I D H$ mutant gliomas, representing a favorable prognostic factor, although this biomarker is not associated with the benefit from either temozolomide or radiotherapy in this molecular subgroup [27].

Currently, these genes are not included in the 2016 WHO classification of diffuse gliomas, although these genetic alterations could be relevant in the diagnostic routine, patient management and on the choice of the treatments $[4,29]$.

In the present study, we aimed to reclassify a 444 cohort of diffused gliomas based on the 2016 WHO classification of Central Nervous System Tumors. Subsequently, we used this reclassified cohort to evaluate the impact of TERT promoter mutations, PTEN deletion, EGFR amplification and MGMT promoter methylation in diagnosis, prognosis and response to therapy.

\section{Material and methods Biological samples}

A dataset of adult diffuse glioma samples was obtained from patients diagnosed from 2011 to 2016, in Unidade de Investigação em Patobiologia Molecular of Instituto Português de Oncologia de Lisboa Francisco Gentil (IPOLFG). In this study we included all consecutive glioma patients referred for treatment in our center, previously submitted to surgery, with known histologic diagnosis and biological material available. This study was previously approved by the IPOLFG Ethical Board Committee. Four hundred forty-four glioma samples were reclassified according to the 2016 WHO classification. However, statistical analysis was performed using only 403 samples, due to the exclusion of the NOS (Not Otherwise Specified) glioma group. These samples were previously characterized in the diagnostic routine for: IDH mutations and TERT promoter mutations by Sanger Sequencing. MGMT promoter methylation was determined by Multiplex Ligation-dependent Probe Amplification (MLPA - MRC-Holland according with guidelines defined by van den Bent [30]). PTEN deletion (Vysis PTEN/CEP10), EGFR amplification (Vysis EGFR/ CEP7) and 1p/19q codeletion (Vysis,1p36/1q25 and 19q13/19.13 dual color probe) were identified by Fluorescent in situ hybridization (FISH). The definition of numerical alterations was performed according the FISH criteria defined by the International System of Human Cytogenetic Nomenclature (ISCN) 2016 [31].

\section{DNA extraction}

For samples without the mutational status of IDH1 ( $n=$ 92) and TERT promoter mutations $(n=82)$, we extracted DNA, when not available from the routine diagnosis, 
which was used at a concentration of $80 \mathrm{ng} / \mu \mathrm{l}$. Tumor samples were received as fresh tissue or paraffinpreserved tissue for DNA extraction. The tumor sections for DNA extraction were selected by a neuropathologist consider the following criteria: 1) cellular regions without necrosis, 2) representative regions of tumor subtype and 3) areas with a minimum of $2 \mathrm{~mm}$ of diameter. The DNA extraction from frozen tissues was performed using the conventional method of phenol-chloroform (MERCK, Germany). From tissues fixed in formaldehyde and preserved in paraffin, the DNA was isolated using the QIAGEN's Gene Read ${ }^{\text {mo }}$ DNA FFPE Kit. Additionally, for some samples included in this project the DNA was extracted using an automatized process by Maxwell ${ }^{\circ}$ RSC Instrument (Promega, USA), using the RSC DNA FFPE kit (Promega, USA). The extraction was performed according to the manufacture's protocol. The DNA concentration and quality were assessed using Nanodrop 2000 (Thermo Fisher Scientific, USA).

\section{Polymerase chain reaction (PCR) and sequencing}

The mutational analysis directed to exon 4 of $I D H 1$ and TERT promoter was performed using two sets of primers for the detection of hotspot mutations: missense mutations involving a single amino acid change at arginine 132 (R132) of IDH1 and C228T and C250T map - 124 and - 146 bp upstream of TERT ATG site. The target amplification of IDH1 was achieved using the forward primer 5' CGGTCT TCAGAGAAGCCATT $3^{\prime}$ and the reverse primer $5^{\prime}$ GCAAAATCACATTATTGCCAAC3' and TERT promoter was amplified using the forward primer 5' GCACAGACGCCCAGGACCGCGCT 3 ' and the reverse primer 5' TTCCCACGTGCGCAGCAGGACGCA 3' generating fragments with $129 \mathrm{bp}$ and $196 \mathrm{bp}$ respectively. PCR contained 35 cycles with annealing at $56^{\circ} \mathrm{C}$ for $I D H 1$ and $69.5^{\circ} \mathrm{C}$ for TERT promoter. Then, an enzymatic method was used to purify each PCR product, using two distinct enzymes: Exonuclease I $20 \mathrm{U} / \mu \mathrm{l}$ (Thermo Fisher Scientific, USA) and FastAP Thermosensitive Alkaline Phosphatase 1 $\mathrm{U} / \mu \mathrm{l}$ (Thermo Fisher Scientific, USA). To determine the sequence of interest in IDH1 and TERT promoter gene, an automatic sequencer was used, ABI PrismTM 3130 Genetic Analyser (Applied Biosystems, USA) following the protocol purposed by Big Dye ${ }^{\text {ma }}$ Terminator v1.1 Cycle Sequencing Kit (Applied Biosystems, USA).

\section{Statistical analysis}

The primary endpoint was overall survival, defined as the time from the glioma diagnosis to the patient death or last follow up. Survival analysis was done using Kaplan-Meier estimator and the log-rank test for group comparison. Variables with a significant $p$-value in the univariate analysis were exposed to a multivariate analysis using Cox regression proportional hazard model.
The multivariate analysis allowed to study the independent association of the molecular subgroups established with overall survival, while controlling for potential confounders such as age, sex and treatment. In order to eliminate confounder variables, the number of cases of each subtype was reduced because the type of treatment was not accessible for all the cases included in the cohort. Additionally, to evaluate the association between the interest biomarkers and the overall survival was performed a multivariate analysis controlling for: $M G M T$ methylation, PTEN deletion and EGFR amplification. $T E R T$ promoter mutations were excluded from this analysis, since the number of samples would reduce the dataset available to determine the impact of the remaining biomarkers.

All tests were two-sided, and we considered a significance level of 5\%. The statistical analysis applied here was performed using IBM SPSS Statistics 21.0.

\section{Results}

The impact of 2016 WHO classification in the stratification of diffuse gliomas

The reorganization of diffuse gliomas according to 2016 WHO classification mainly affected oligodendroglioma and astrocytoma subgroups, reducing the number of oligodendrogliomas (82 to 49) and increasing the astrocytomas (49 to 98), while the number of GBM remained the same (Table 1). Additionally, 41 samples were not included in any glioma subset (Glioma NOS), mainly due to: technical issues, samples with only $1 \mathrm{p}$ or $19 \mathrm{q}$ deletion or with $1 \mathrm{p} / 19 \mathrm{q}$ codeletion and $I D H$-wildtype. Importantly, in this new classification the subgroup of oligoastrocytomas was reorganized between astrocytomas, oligodendrogliomas or NOS.

This molecular reclassification allowed the division of diffuse gliomas into 6 molecular subgroups according to $I D H$ mutations and $1 \mathrm{p} / 19 \mathrm{q}$ codeletion analysis (Table 2). GBM samples corresponded to $57.7 \%$ of the entire cohort, of which $55.2 \%$ were GBM $I D H$ wildtype and the remaining $2.5 \%$ were GBM $I D H$-mutant. The astrocytoma subgroup was the second most frequent $(22.1 \%)$ and the NOS glioma subgroup corresponded to $9.2 \%$ of the cohort analyzed (Table 2). $I D H$-mutant gliomas, whether GBMs or astrocytomas, are predominant in young patients (44 and 38 years respectively) in comparison with $I D H$-wildtype gliomas (63 and 57 years respectively) (Table 3). In addition, our results also indicated a higher prevalence of GBM $I D H$-wildtype, astrocytomas $I D H$-wildtype and $I D H$-mutant and $1 \mathrm{p} / 19$ codeleted gliomas in men $(2: 1 ; 1.3: 1 ; 1.4: 1$ and 2.1 respectively), except for the GBM $I D H$-mutant subgroup (0.8:1) (Table 3).

Then, we evaluated the prognostic value of histological grade and molecular subgroups. Grade II 
Table 1 Effect of 2016 WHO classification on the subdivision of glioma subgroups

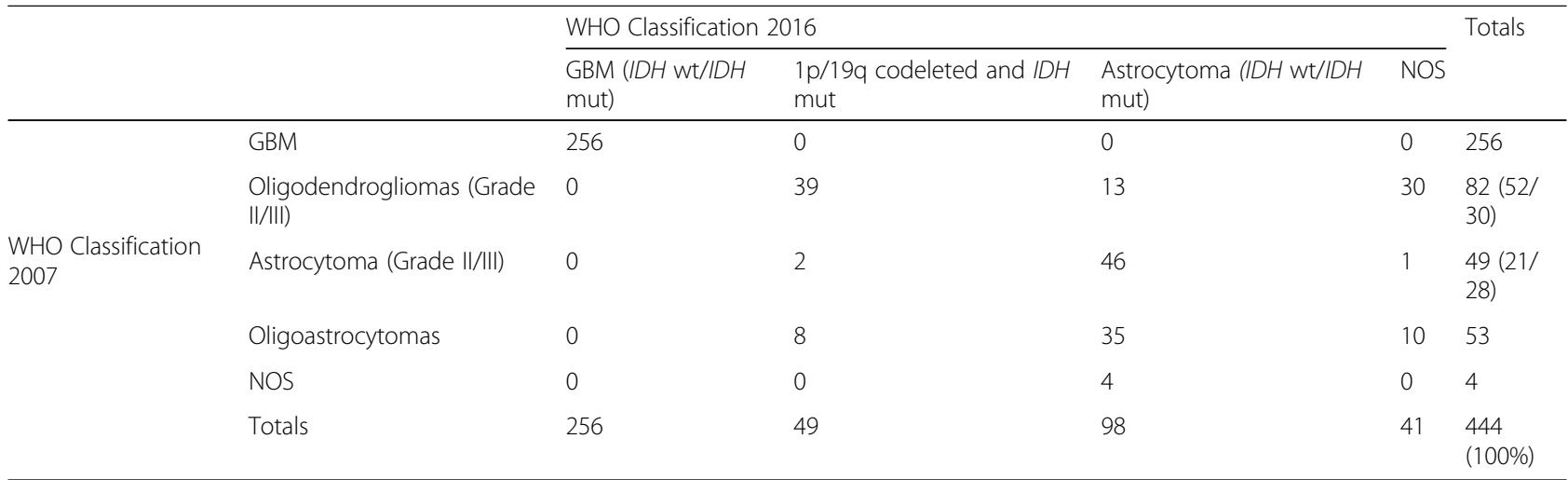

oligodendroglioma was the histological subgroup associated with longer overall survival (Median Overall Survival (OS):172 months); in contrast, GBM (grade IV) was the subgroup associated with the poorer outcome (OS:11 months) (Fig. 1). Using the molecular classification, gliomas with $1 \mathrm{p} / 19 \mathrm{q}$ codeletion were the subgroup associated with better prognosis (OS:198 months). For the remaining molecular subgroups (GBM and astrocytomas), IDH-mutant tumors were associated with better prognosis (OS:25 and 114 months, respectively) when compared to the $I D H$-wildtype subgroups (OS:10 and 14 months, respectively) (Fig. 1).

The multivariate analysis performed using Cox Regression Hazard model evidenced the prognostic impact of the molecular subgroups and histological grades, after adjustment for age, gender and treatment (Fig. 1). The GBM IDH-mutant subgroup was the only group that did not show a statistically significant $p$-value $(p$-value $=0.092)$, perhaps due to the reduced number of samples $(n=10)$. These results validated the size and representativeness of each glioma molecular subgroup to perform further studies, as well as, the accuracy introduced by molecular

Table 2 Relative frequency of glioma molecular subgroups according to the 2016 WHO classification

\begin{tabular}{lll}
\hline Molecular Subgroups & Number of samples & $\%$ Gliomas \\
\hline GBM, IDH mutant & 11 & $2.5(11 / 444)$ \\
GBM, IDH-wildtype & 245 & $55.2(245 / 444)$ \\
Astrocytoma, IDH-mutant & 55 & $12.4(55 / 444)$ \\
Astrocytoma, IDH-wildtype & 43 & $9.7(43 / 444)$ \\
IDH mutant and 1p/19q codeleted & 49 & $11.0(49 / 444)$ \\
NOS & 41 & $9.2(41 / 444)$ \\
Total & 444 & $100(444 / 444)$ \\
\hline
\end{tabular}

markers in the prognosis and diagnosis of patients with gliomas.

\section{The frequency of TERT promoter mutations, EGFR amplification, PTEN deletion and MGMT promoter methylation in molecular glioma subgroups}

Following the reclassification of gliomas according to the 2016 WHO classification, we investigated the role of TERT promoter mutations, EGFR amplification, PTEN deletion and MGMT promoter methylation in molecular glioma subgroups. Here, we intended to assess whether these molecular alterations are predominantly altered in a specific subgroup, which could help to redefine the established molecular subgroups of gliomas. EGFR amplification was more frequently detected in $I D H$-wildtype gliomas, both, GBM (38\%) and astrocytomas (43\%), compared to $I D H$-mutant gliomas (11 and $4 \%$, respectively). This molecular alteration was absent from the $1 \mathrm{p} /$ $19 \mathrm{q}$ codeleted glioma subgroup (0\%). PTEN deletions were identified in $83 \%$ of GBM IDH-wildtype, the most aggressive glioma group (Fig. 2), characterized by an OS of 10 months (Fig. 1). However, these alterations were also found in 43 and 50\% of GBM IDH-mutant (OS:25 months) and astrocytomas $I D H$-wildtype (OS:14 months) respectively. In 1p/19q codeleted gliomas and astrocytomas $I D H$-mutant (OS:198 and 114 months, respectively), the two less aggressive subtypes of gliomas, the incidence of PTEN deletion was reduced (8 and 21\% respectively). These results suggested that PTEN deletions are predominantly found in the most aggressive subgroups of gliomas. TERT promoter mutations were mainly found in 1p/19q codeleted (94\%) and GBM IDHwildtype (88\%) molecular subgroups (Fig. 2), suggesting that this is not a good biomarker for diagnosis. Regarding MGMT promoter methylation status, we observed that: $100 \%$ of $1 \mathrm{p} / 19 \mathrm{q}$ codeleted gliomas, $91 \%$ of astrocytomas IDH-mutant and 50\% of GBM IDH-mutant samples were methylated. Therefore, MGMT promoter methylation, as expected, was inversely associated with 
Table 3 Clinicopathological data of gliomas patients based on the molecular subgroups

\begin{tabular}{|c|c|c|c|c|c|c|}
\hline \multirow[t]{2}{*}{ Variable } & \multirow[t]{2}{*}{ No } & \multicolumn{5}{|c|}{ Glioma Molecular subgroups } \\
\hline & & $\begin{array}{l}\text { GBM, IDH } \\
\text { wildtype }\end{array}$ & $\begin{array}{l}\text { GBM, IDH } \\
\text { mutant }\end{array}$ & $\begin{array}{l}1 p / 19 q \text { codeleted } \\
\text { gliomas }\end{array}$ & $\begin{array}{l}\text { Astrocytoma, IDH } \\
\text { wildtype }\end{array}$ & $\begin{array}{l}\text { Astrocytoma, IDH } \\
\text { mutant }\end{array}$ \\
\hline Number of samples & 403 & 245 & 11 & 49 & 43 & 55 \\
\hline \multirow{3}{*}{$\begin{array}{l}\text { Age of diagnosis } \\
\text { (years) }\end{array}$} & Median & 63.0 & 44.0 & 48.5 & 57.0 & 38.0 \\
\hline & Minimum & 18.0 & 17.0 & 27.0 & 16.0 & 23.0 \\
\hline & Maximum & 87.0 & 59.0 & 76.0 & 80.0 & 66.0 \\
\hline \multirow[t]{3}{*}{ Sex } & Male & 163 & 5 & 34 & 24 & 32 \\
\hline & Female & 82 & 6 & 15 & 19 & 23 \\
\hline & $\begin{array}{l}\text { Ratio (M/ } \\
\text { F) }\end{array}$ & $2: 1$ & $0.8: 1$ & $2: 1$ & 1.3:1 & 1.4:1 \\
\hline \multirow[t]{3}{*}{ Adjuvant Therapy } & Receveid & 211 & 11 & 30 & 27 & 45 \\
\hline & None & 19 & 0 & 1 & 6 & 3 \\
\hline & No data & 15 & 0 & 18 & 10 & 7 \\
\hline
\end{tabular}

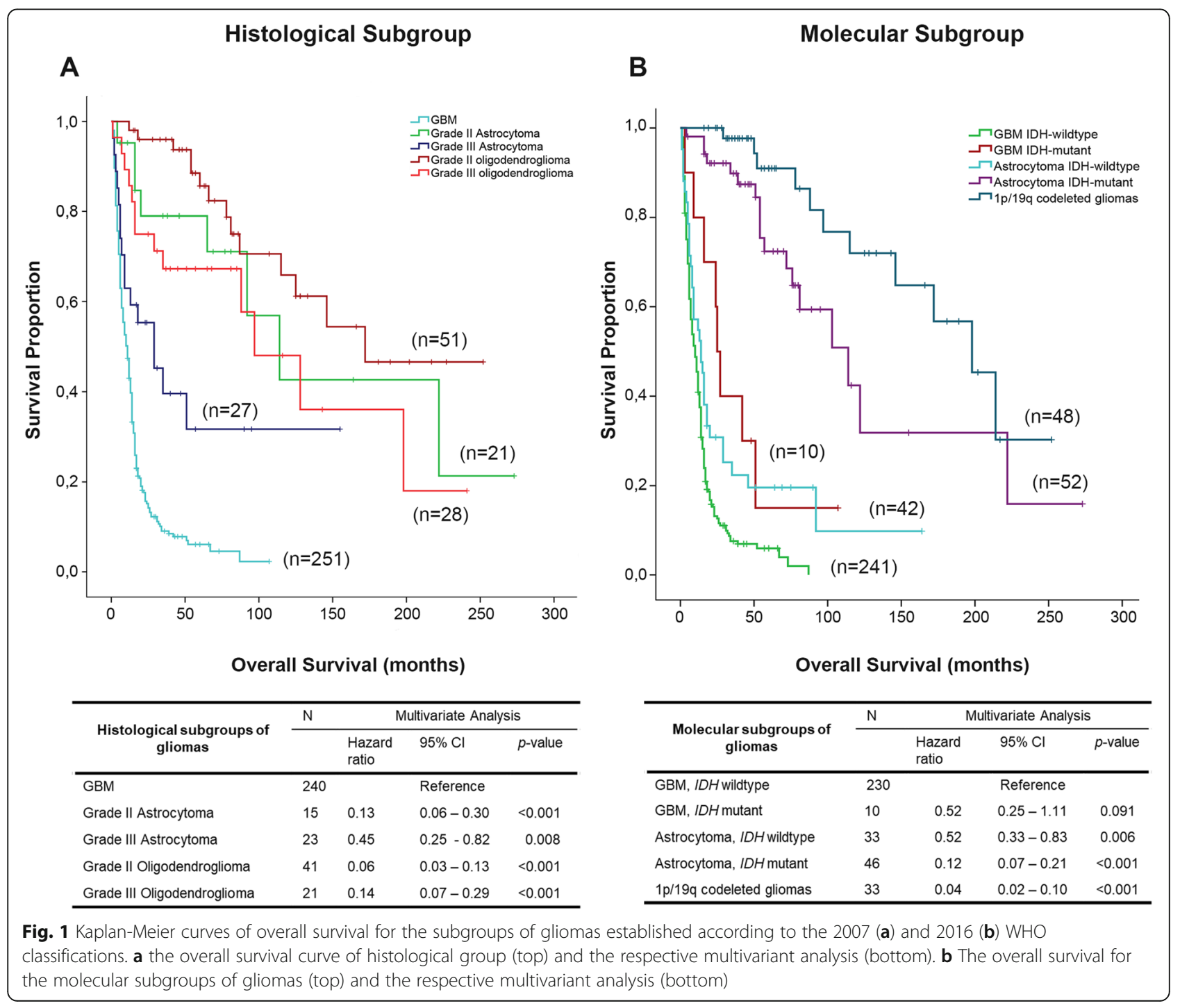




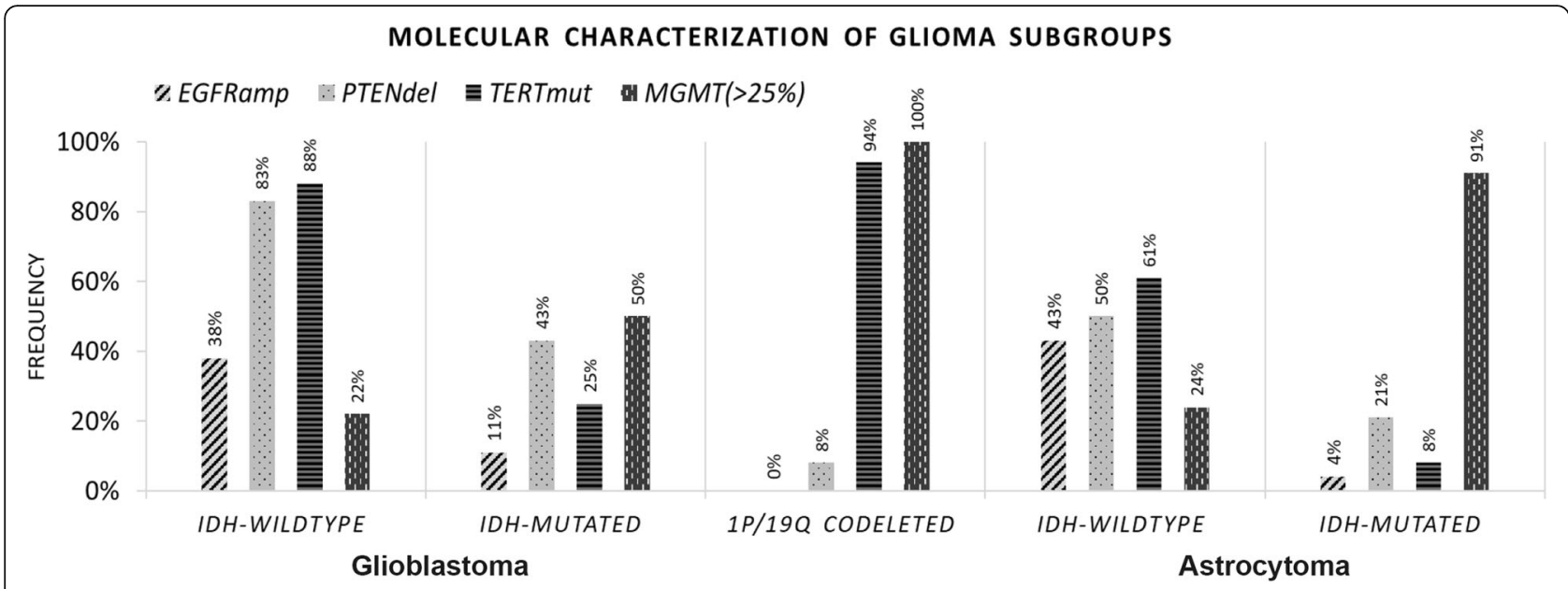

Fig. 2 Frequency of EGFR amplification (amp), PTEN deletion (del), TERT promoter mutations (mut) and MGMT promoter methylated samples in the distinct glioma molecular subgroups. EGFR amplification was analyzed: in 227 GBM-IDH wildtype, 9 GBM IDH-mutant, 48 1p/19q codeleted gliomas, 35 Astrocytoma IDH-wildtype, 53 Astrocytomas IDH-mutant. PTEN deletion was analyzed: in 225 GBM IDH-wildtype, 7 GBM IDH-mutant, 48 1p/19q codeleted gliomas, 37 Astrocytoma IDH-wildtype and 53 Astrocytoma IDH-mutant. TERT promoter mutations were analyzed: in 124 GBM IDH-wildtype, 4 GBM IDH-mutant, 49 1p/19q codeleted gliomas, 41 Astrocytoma IDH-wildtype, 51 Astrocytoma IDH-mutant. MGMT methylation was analyzed: in 235 GBM IDH-wildtype, $10 \mathrm{GBM}$ IDH-mutant, 49 1p/19q codeleted gliomas, 41 Astrocytoma IDH-wildtype and 54 Astrocytoma IDH-mutant

aggressiveness, since it appears most frequently in groups with better prognosis (Fig. 2).

\section{Prognostic impact of EGFR amplification, PTEN deletion, TERT promoter mutations and MGMT promoter methylation}

Furthermore, we evaluated the prognostic value of these distinct genetic alterations in each molecular subgroup of gliomas. We verified that EGFR amplification did not have significant impact in the overall survival of patients with GBM $I D H$-wildtype and astrocytoma $I D H$-wildtype, ( $p=0.393$ and $p=0.522$, respectively) (Fig. $3 \mathrm{a}$ and b), as well as, TERT promoter mutations in GBM IDH-wildtype $(p=0.605)$, although the number of cases was too small for conclusive results (Fig. 3c).

However, as shown in Fig. 3d, MGMT promoter methylation was significantly associated with a prolonged overall survival in GBM IDH-wildtype ( $p=$ 0.009), while in astrocytomas $I D H$-wildtype (Fig. 3e) had no impact in prognosis $(p=0.555)$. The effect of this biomarker in astrocytomas $I D H$-wildtype could be related with the low number of methylated samples $(n=$ 9).

Surprisingly, PTEN deletion had a dual effect in the prognosis of GBM and Astrocytomas $I D H$-wildtype. This molecular alteration was a favorable prognostic for GBM $I D H$-wildtype $(p=0.042)$ and a unfavourable prognostic for astrocytoma $I D H$-wildtype $(p=0.015)$ (Fig. 3f and g). Moreover, in astrocytomas IDH-mutant, PTEN deletion was not found to have a significant impact on overall survival $(p=0.702)$ (Fig. 3h). The multivariate analysis, considering EGFR amplification, PTEN deletion and MGMT methylation and controlling for age, gender and treatment, validated the role of PTEN deletion (Hazard Ratio (HR) $=0.65$; 95\% CI 0.43-0.99) and MGMT promoter methylation $(\mathrm{HR}=0.61 ; 95 \% \mathrm{CI} 0.42-0.88)$ as independent factors of prognosis in GBM $I D H$-wildtype. In addition, also confirmed the role of PTEN deletion as a prognostic factor of poor outcome $(\mathrm{HR}=4.48 ; 95 \% \mathrm{CI}$ 1.34-14.94) (Table 4).

The predictive effect of EGFR amplification, PTEN deletion and MGMT promoter methylation in GBM IDH-wildtype patients

To gain further insight into the predictive value of these biomarkers in molecular subgroups of gliomas, we analyzed the effect of EGFR amplification, PTEN deletion and $M G M T$ promoter methylation in the response to therapy using the only group with representative samples - GBM IDH-wildtype (Fig. 4). However, in this group due to the small number of TERT wildtype samples, it was not possible to do this analysis regardless TERT promoter mutations.

Initially, we analyzed the OS of each group of patients treated with radiotherapy (RT) or chemo-radiotherapy (CRT), which was 6 and 16 months, respectively (Fig. 4a). CRT based on temozolomide is the standard treatment for patients with GBM. Patients subjected to RT alone, usually respect the following criteria: age above 70 years, other pathological conditions contra-indicating chemotherapy or a more severe clinical presentation. 

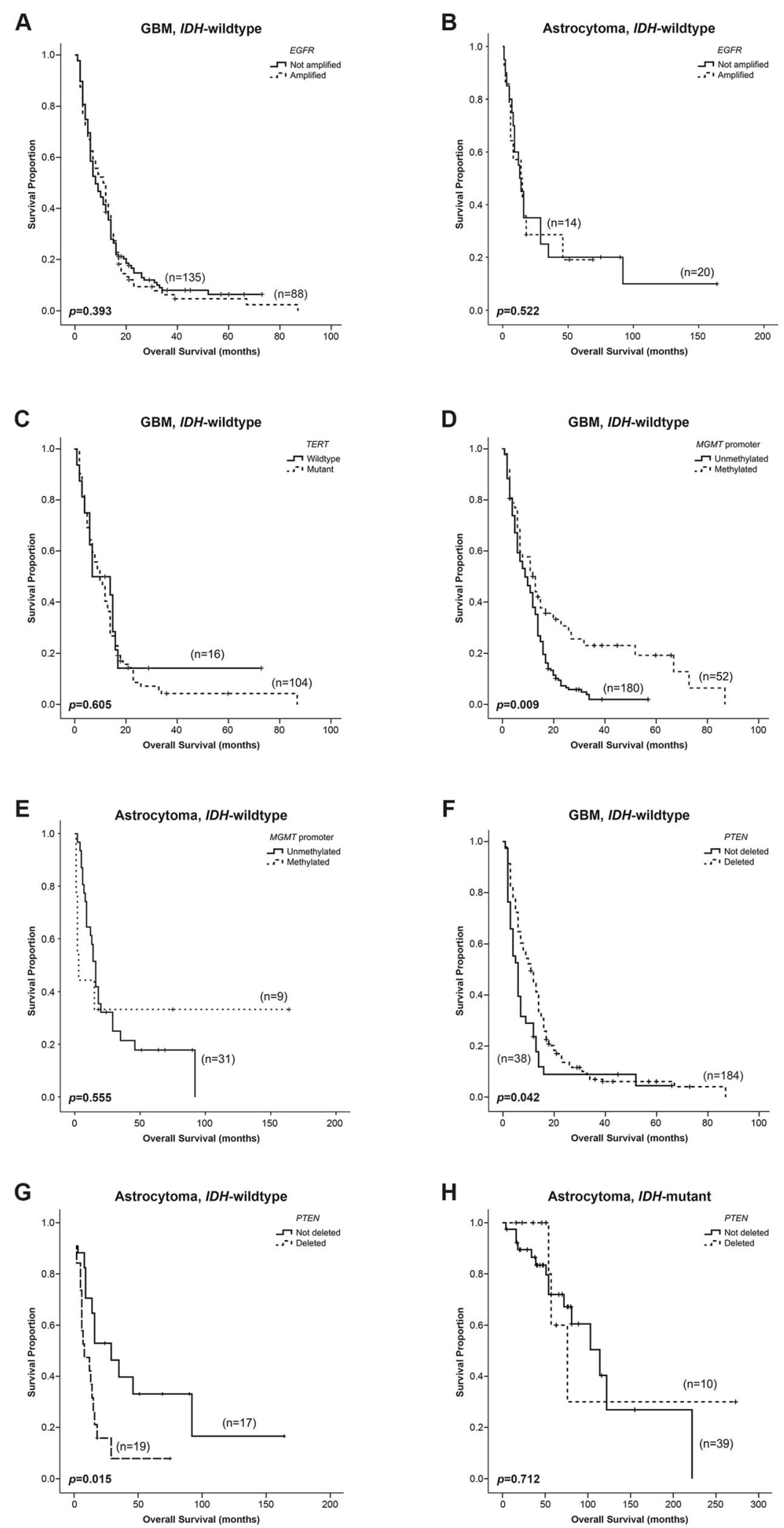

Fig. 3 (See legend on next page.) 
(See figure on previous page.)

Fig. 3 The impact of EGFR amplification, TERT promoter mutations, PTEN deletion and MGMT promoter methylation on overall survival of glioma patients. Kaplan-Meier curves of EGFR amplification in GBM IDH-wildtype (a) and in astrocytoma IDH-wildtype (b). Kaplan-Meier curves of TERT promoter mutations (c) and MGMT promoter methylation in GBM IDH-wildtype (d) and in astrocytoma IDH-wildtype (e). The impact of PTEN deletion in overall survival of GBM IDH-wildtype (f), astrocytomas IDH-wildtype $(\mathbf{g})$ and IDH-mutant (h)

Patients who were not treated with RT or CRT, were directly to palliative care.

In patients with GBM $I D H$-wildtype, surprisingly the EGFR amplification was associated with a better response to radiotherapy ( $p=0.011)$ (Fig. 4b left), however it was unable to predict the response to chemoradiotherapy ( $p=0.596$ ) (Fig. $4 \mathrm{c}$ right). The multivariate analysis performed in RT subgroup, considering the three genetic alterations and controlling for age and gender revealed that EGFR amplification constitutes an independent predictive factor of response to radiotherapy (HR $=0.56$; 95\% CI 0.36-0.88) (Table 5). This result suggests a new putative strategy for the management of patients, who may have a better response to radiotherapy, although it should be validated in other cohorts.

The effect of PTEN deletion on response to therapy was inferred only in patients exposed to radiotherapy, since most patients submitted to chemo-radiotherapy

Table 4 Multivariate analysis for the prognostic impact of IDH mutations, PTEN deletion, MGMT methylation and EGFR amplification

\begin{tabular}{|c|c|c|c|c|c|c|}
\hline & \multirow[t]{2}{*}{$\mathrm{N}$} & \multirow{2}{*}{$\begin{array}{l}\text { Median } \\
\text { Survival } \\
\text { (MS- } \\
\text { months) }\end{array}$} & \multirow[t]{2}{*}{$\mathrm{N}$} & \multicolumn{3}{|c|}{ Multivariate analysis $^{a}$} \\
\hline & & & & Hazard ratio & $95 \% \mathrm{Cl}$ & $\overline{p \text {-Value }}$ \\
\hline \multicolumn{7}{|l|}{$\overline{\text { GBM }}$} \\
\hline \multicolumn{7}{|l|}{$I D H$} \\
\hline Mutant & 10 & 25.0 & 10 & 0.52 & $0.24-1.11$ & 0.092 \\
\hline Wildtype & 241 & 10.0 & 230 & & Reference & \\
\hline \multicolumn{7}{|l|}{ Astrocytoma } \\
\hline \multicolumn{7}{|l|}{$I D H$} \\
\hline Mutant & 42 & 14.0 & 33 & 0.22 & $0.11-0.43$ & $<0.001$ \\
\hline Wildtype & 52 & 114.0 & 46 & & Reference & \\
\hline \multicolumn{7}{|l|}{ GBM IDH-wildtype } \\
\hline \multicolumn{7}{|l|}{ PTEN } \\
\hline Deleted & 184 & 11.0 & 171 & 0.65 & $0.43-0.99$ & 0.042 \\
\hline Non-Deleted & 38 & 6.0 & 34 & & Reference & \\
\hline \multicolumn{7}{|l|}{ MGMT } \\
\hline Methylated & 52 & 12.0 & 46 & 0.61 & $0.42-0.88$ & 0.009 \\
\hline Unmethylated & 180 & 9.0 & 159 & & Reference & \\
\hline \multicolumn{7}{|l|}{ EGFR } \\
\hline Amplified & 88 & 11.0 & 82 & 0.88 & $0.65-1.18$ & 0.393 \\
\hline Non- Amplified & 135 & 8.0 & 123 & & Reference & \\
\hline \multicolumn{7}{|c|}{ Astrocytoma IDH-wildtype } \\
\hline \multicolumn{7}{|l|}{ PTEN } \\
\hline Deleted & 18 & 8.0 & 14 & 4.48 & $1.34-14.94$ & 0.015 \\
\hline Non-deleted & 17 & 29.0 & 11 & & Reference & \\
\hline \multicolumn{7}{|l|}{ MGMT } \\
\hline Methylated & 9 & 3.0 & 7 & 0.69 & $0.20-2.4$ & 0.555 \\
\hline Unmethylated & 31 & 16.0 & 18 & & Reference & \\
\hline \multicolumn{7}{|l|}{ EGFR } \\
\hline Amplified & 14 & 14.0 & 12 & 0.71 & $0.25-2.03$ & 0.522 \\
\hline Non- Amplified & 20 & 13.0 & 13 & & Reference & \\
\hline
\end{tabular}

${ }^{\mathrm{a}}$ Multivariate analysis was performed controlling the following independent variables: age, gender, treatment 


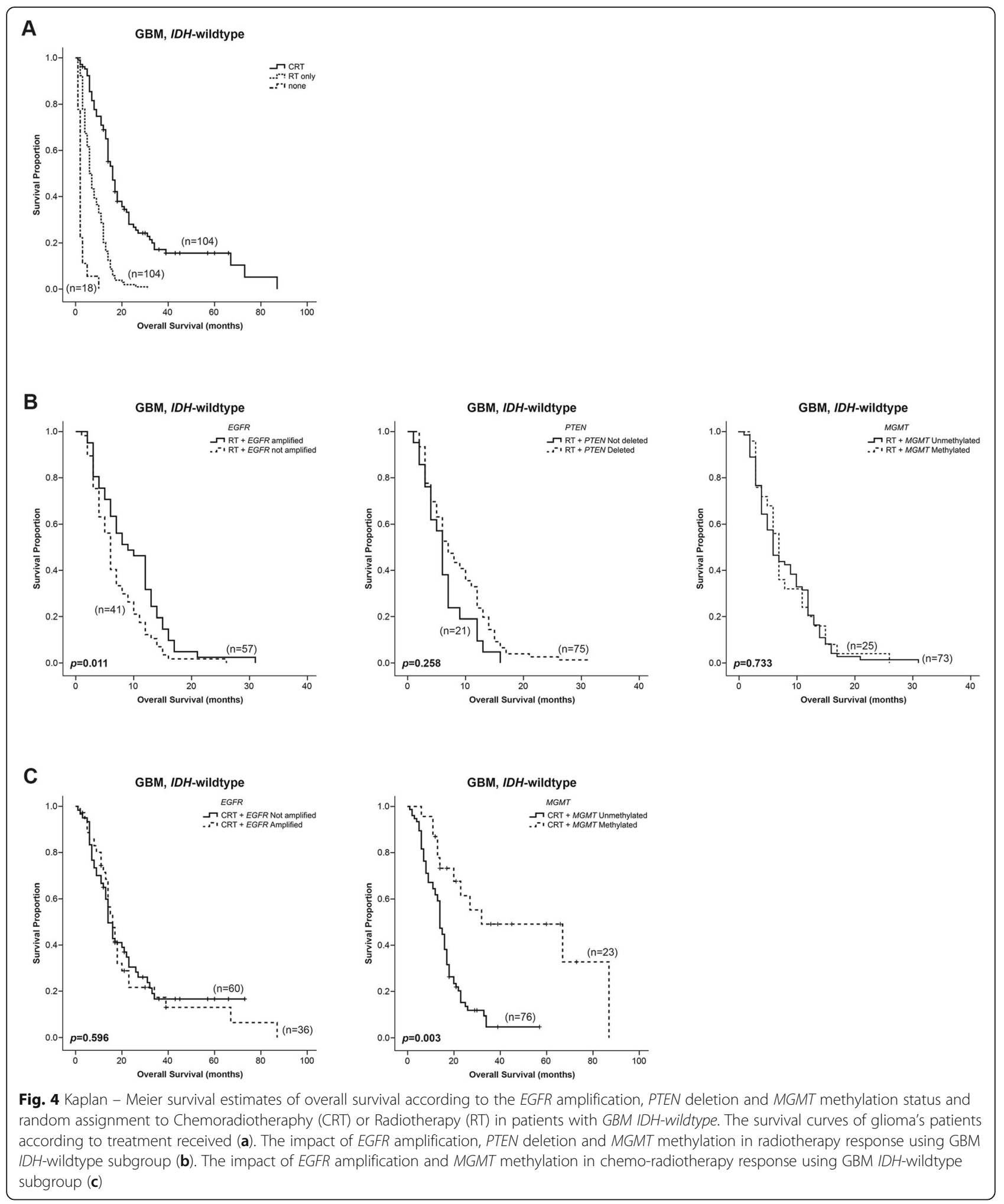

had PTEN deleted. According to our results, PTEN deletion had no predictive value in the response to radiotherapy $(p=0.258)$ (Fig. 4b middle).
Additionally, our results showed that MGMT methylated samples were associated with an improved response to chemo-radiotherapy compared to MGMT 
Table 5 Multivariate analysis for the predictive value of PTEN deletion, MGMT methylation and EGFR amplification in GBM IDH wildtype

\begin{tabular}{|c|c|c|c|c|c|}
\hline & \multirow{2}{*}{$\begin{array}{l}\text { Median } \\
\text { Survival } \\
\text { (MS- } \\
\text { months) }\end{array}$} & \multirow[t]{2}{*}{$\mathrm{N}$} & \multicolumn{3}{|c|}{ Multivariate analysis ${ }^{a}$} \\
\hline & & & Hazard ratio & $95 \% \mathrm{Cl}$ & $p$-Value \\
\hline \multicolumn{6}{|l|}{ GBM IDH-wildtype } \\
\hline CRT & 16.0 & 104 & 0.053 & $0.029-0.098$ & $<0.001$ \\
\hline RT & 6.0 & 104 & 0.181 & $0.105-0.314$ & $<0.001$ \\
\hline None & 2.0 & 18 & & Reference & \\
\hline \multicolumn{6}{|l|}{ RT } \\
\hline \multicolumn{6}{|l|}{ PTEN } \\
\hline Deleted & 7.0 & 74 & 0.74 & $0.45-1.24$ & 0.258 \\
\hline Non-deleted & 6.0 & 20 & & Reference & \\
\hline \multicolumn{6}{|l|}{ MGMT } \\
\hline Methylated & 7.0 & 24 & 1.09 & $0.67-1.78$ & 0.733 \\
\hline Unmethylated & 6.0 & 70 & & Reference & \\
\hline \multicolumn{6}{|l|}{ EGFR } \\
\hline Amplified & 9.0 & 39 & 0.56 & $0.36-0.88$ & 0.011 \\
\hline Non-amplified & 6.0 & 55 & & Reference & \\
\hline \multicolumn{6}{|l|}{ CRT } \\
\hline \multicolumn{6}{|l|}{ PTEN } \\
\hline Deleted & 14.0 & 84 & 1.68 & $0.40-7.15$ & 0.481 \\
\hline Non-deleted & 14.0 & 5 & & Reference & \\
\hline \multicolumn{6}{|l|}{ MGMT } \\
\hline Methylated & 32.0 & 20 & 0.34 & $0.17-0.69$ & 0.003 \\
\hline Unmethylated & 14.0 & 69 & & Reference & \\
\hline \multicolumn{6}{|l|}{ EGFR } \\
\hline Amplified & 15.0 & 32 & 0.88 & $0.54-1.43$ & 0.596 \\
\hline Non-amplified & 14.0 & 57 & & Reference & \\
\hline
\end{tabular}

${ }^{\mathrm{a}}$ Multivariate analysis was performed controlling the following independent variables: age and gender. $R T$ Radiotherapy, CRT Chemo-radiotherapy

unmethylated samples in GBM $I D H$-wildtype patients ( $p=0.003)$ (Fig. 4c right). MGMT methylation constitutes a well-known predictive biomarker of gliomas used to infer which patients would have a better response to chemotherapy with temozolomide. Despite of its effects in response to chemo-radiotherapy, as described before [25], MGMT was not an important predictor of response to radiotherapy alone $(p=0.733)$ (Fig. 4b right).

\section{Discussion}

In the present study we evaluated the impact of the new 2016 WHO classification of Central Nervous System Tumors in a 444 diffuse gliomas cohort, previously classified according to the 2007 WHO classification based on histological features.
Our results showed a decrease in the percentage of oligodendrogliomas, from $18.5 \%$ of the samples previously diagnosed using the histological classification, to $11 \%$ of the samples according to the new classification. On the other hand, there was an increase in the percentage of astrocytomas (from 11 to $22.1 \%$ of the samples). This main alteration in glioma subgroups was associated with the introduction of $1 \mathrm{p} / 19 \mathrm{q}$ codeletion and $I D H$ status, which were decisive in the subdivision of astrocytoma and oligodendroglioma as well as the disintegration of the oligoastrocytoma group. These results are in accordance with the study of Iuchi et al., which reported astrocytoma and oligodendroglioma subgroups as the main targets of the 2016 WHO classification effect [32]. However, according to Tabouret and co-authors, the reclassification of the French cohort showed a similar frequency of oligodendrogliomas before and after the reclassification of gliomas (31.6-34.5\%, respectively), while the number of GBM (33.8-50.3\%) and astrocytomas (7-16.2\%) increased [33]. The differences observed between our study and the French cohort, could be related with the reclassification of oligoastrocytomas, since in our study most oligoastrocytomas were reclassified as astrocytoma $(n=35)$, while in the study of Tabouret et al. the vast majority of oligoastrocytomas were considered GBM [33].

Even with the introduction of molecular biomarkers, the distribution of patients previously diagnosed with oligoastrocytomas remains a difficult task, which is demonstrated by the variability between studies [32,33]. In our study 10 samples of oligoastrocytomas were included into the NOS subgroup. The analysis of alpha thalassemia/mental retardation syndrome $\mathrm{X}$-linked (ATRX) loss and tumor protein 53 (TP53) mutations was not performed, which constitutes a limitation of this study. The analysis of these both biomarkers is suggested in the 2016 WHO classification only in doubtful cases [4], and they are currently done in our Institute by immunochemistry. Actually, the mutational status of these genes is not determined in diagnosis of gliomas for two main reasons: i) by itself are unable to identify the subtype of glioma sample and because; ii) it is expensive, they constitute long genes, becoming difficult their analysis using the conventional molecular techniques.

In total, 41 samples of our cohort were inserted into the NOS glioma subgroup, highlighting the need for new biomarkers, in order to be possible to classify gliomas with $1 \mathrm{p}$ or $19 \mathrm{q}$ deletion and gliomas $I D H$-wildtype with $1 \mathrm{p} / 19 \mathrm{q}$ codeletion. Although, it is important to note the most of them are included in this subgroup due to technical issues.

Here, as expected, GBM constituted the most prevalent type of glioma (57.7\%), like previously reported by Iuchi et al. (66\%), Tabouret et al. (50\%) and Ostrom et al. (45\%) [1, 32, 33]. However, GBM IDH-mutant accounted for only $2.5 \%$ of all GBM, slightly less than 
the $10,17.2$ and $7.8 \%$ previously reported [32-34]. In addition, astrocytomas $I D H$-wildtype and $I D H$-mutant showed similar frequencies (9.7 and $12.4 \%$ respectively), slightly different from the results reported by Iuchi and co-authors $(13.7 \%$ of astrocytomas IDH-wildtype and $6.7 \%$ of astrocytomas $I D H$-mutant) and Tabouret et al., (11\% astrocytomas $I D H$-mutant and $5.3 \%$ of astrocytoma $I D H$-wildtype) [32, 33]. The differences detected in astrocytomas diagnosis could be related to their reclassification in GBM, which presently depends only on the histological features of the tumor, introducing some variability between studies.

Notably, 1p/19q codeleted gliomas were the molecular subgroup associated with the longest overall survival (OS: 198 months), regardless of whether they were classified as oligodendrogliomas grade II (OS:172 months) or grade III (OS:97 months). These results suggested that $1 \mathrm{p} / 19 \mathrm{q}$ codeletion is a strong biomarker of prognosis and even better than histological classification, since could embrace less aggressive tumors. In addition, these statements are in accordance with previous studies indicating $1 \mathrm{p} / 19 \mathrm{q}$ codeletion was associated with a better prognosis when compared to non-codeleted tumors $[35,36]$.

Furthermore, $I D H$ mutational analysis divided the astrocytoma group into two subgroups with distinct prognoses $(p<0.001)$, as previously reported [4, 37]. Here, $I D H$ mutations did not had prognostic impact in GBM $(p=0.092)$ (Table 5), which could be explained by the reduced number of GBM $I D H$-mutant samples $(n=11)$.

We also evaluated the impact of EGFR amplification, PTEN deletion, TERT promoter mutations and MGMT promoter methylation in the diagnosis, prognosis and response to therapy of patients with diffuse gliomas. As reported by other studies, EGFR amplification was more common in $I D H$-wildtype gliomas [38-40]. To date, most studies evaluated the prognostic value of EGFR amplification using only the histological diagnosis, instead of the molecular subgroups of gliomas [21-23]. In this work, we reported for the first-time that EGFR amplification had no significant prognostic value in molecular subgroups of gliomas - IDH-wildtype GBM and astrocytomas. Nevertheless, we only evaluated the presence of EGFR amplification and not EGFR activating mutations. Previously, it was described that tumors with both EGFRvIII overexpression and EGFR amplification constitute an indicator of poor prognosis in GBM patients [23]. The prognostic value of EGFR was also associated with patient's age, seeming to be correlated with worse outcomes in younger patients [41]. However, none of these studies considered the mutational status of $I D H$, which means that the effect on prognosis by EGFR amplification may be dependent of $I D H$ mutations.

Additionally, EGFR amplification has been appointed as one of the causes for the development of radio- resistance in gliomas [42]. Most interestingly, we found that patients with GBM IDH-wildtype and EGFR amplification had a significantly better overall survival than those without EGFR amplification, only when treated with radiotherapy alone, and not when treated with chemo-radiotherapy. At this point, the clinical significance of this finding, and the reasons why it did not occur with chemo-radiotherapy, are not fully understood. However, this result should be validated in other cohorts with a higher number of samples. Nevertheless, our observation is consistent with the previously described in non-small cell lung cancer, where the presence of EGFR activating mutations and also EGFR amplification were associated with a radiosensitive phenotype, inducing increased levels of pro-apoptotic proteins and reduced capability to repair DNA [43-45].

The relative frequency and prognostic value of PTEN deletion in diffuse gliomas were analyzed using histological diagnosis, which explains the variability between the reported studies $[16,17]$. Interestingly, the observed role of PTEN deletion in prognosis of GBM and astrocytomas $I D H$-wildtype has never been documented. In our study, PTEN deletion was considered a factor of good prognosis in GBM $I D H$-wildtype $(p=0.042)$, although using a reduced number of samples without PTEN deletion ( $n=38$ vs $n=184$ with PTEN deleted). Despite this, it was previously noticed that PTEN loss could be associated with a more favorable prognosis, since it leads to a better response to chemotherapy by compromising homologous recombination of DNA, through the transcriptional regulation of $\operatorname{Rad} 51[16,46]$. Another hypothesis to the observed result in our study may be the absence of an inverse correlation between PTEN expression and AKT activity, as demonstrated in melanoma and breast cancer $[47,48]$. Moreover, this dual effect of PTEN deletion in prognosis could be related with the specific tyrosine which is the target of PTEN phosphorylation [49]. This hypothesis would explain why PTEN deletion predicts a good outcome in GBM $I D H$-wildtype. In contrast, in astrocytomas $I D H$-wildtype the deletion of PTEN is a factor of poor prognosis, as expected, since this is a tumor suppressor gene. Further work should be undertaken to evaluate the mechanisms through which this molecular alteration differentially affects the prognosis of these both groups of gliomas.

In this dataset, TERT promoter mutations did not have prognostic value in GBM $I D H$-wildtype, which is consistent with Nguyen et al. and Eckel-Passow et al. previous studies $[17,18]$. Eckel-Passow et al. reported that TERT promoter mutations are associated with a poor prognosis in the absence of $I D H$ mutations in grade II and III gliomas [18]. Therefore, this study verified that TERT promoter mutations would be an important biomarker in grade II and III gliomas. However, these results are not 
according to the 2016 WHO classification. The authors were not considering a subgroup of $1 \mathrm{p} / 19 \mathrm{q}$ codeletion and $I D H$ mutation, as an independent group [18]. Interestingly in our results $1 \mathrm{p} / 19 \mathrm{q}$ codeleted gliomas showed a higher percentage of TERT promoter mutations - $94 \%$. Therefore, this suggests that the effect observed on overall survival by Eckel-Passow et al., could be associated with the difference between $1 \mathrm{p} / 19 \mathrm{q}$ codeleted/IDH mutant gliomas and astrocytomas independently of TERT promoter mutations.

MGMT promoter methylation is a biomarker extensively studied in GBM. As previously mentioned, this biomarker is a prognostic factor of prolonged overall survival $[24,26]$. Here, MGMT methylation was found mainly in $I D H$-mutant gliomas, which is in accordance with the literature [3, 27]. $I D H$ mutations are responsible for increased levels of 2-hydroxiglutarate, which in turn determines the inhibition of several enzymes, such as Jumonji-C domain-containing histone lysine demethylases [50]. It is already known that MGMT methylation is associated with better outcomes in both $I D H$ mutant and $I D H$ wildtype GBM $[27,28]$, although it only constitutes a predictive biomarker for the benefit to temozolomide chemotherapy and not to radiotherapy in patients with GBM-IDH wildtype [25]. Interestingly, Rivera et al., reported the predictive value of $M G M T$ methylation to radiotherapy response in GBM patients, independently of the $I D H$ mutational status [51].

\section{Conclusions}

In this work we demonstrated that the 2016 WHO classification brought an improvement in the accuracy of diagnosis and prognosis of diffuse gliomas, validating the importance of adding molecular characteristics to histology. However, this new classification has limitations to stratify these heterogeneous tumors, for instance, GBM $I D H$-wildtype subgroup had a higher disparity in patients' survival as well as astrocytomas $I D H$ wildtype. Another important issue is gliomas NOS, which embrace gliomas with $1 \mathrm{p} / 19 \mathrm{q}$ codeleted but $I D H$ wildtype or even tumors with partial deletion (1p or 19q). Furthermore, this study highlighted the clinical importance of gathering additional biomarkers in the diagnostic routine of gliomas. TERT promoter mutations seem not to confer additional information about gliomas diagnosis and prognosis. In GBM IDH-wildtype molecular subgroup, PTEN deletion seems to be important for prognosis and $E G F R$ amplification for radiotherapy response. In astrocytoma $I D H$-wildtype, PTEN deletion appear to be important in prognosis. These interesting findings should be validated in other cohorts as well as the in vitro studies should be performed to clarify the molecular mechanisms behind this biological behavior. In contrast, MGMT promoter methylation has been shown to be a strong biomarker of prognosis and a predictor of response to chemotherapy, reinforcing the idea that this biomarker should be include in glioma classification, resulting in a new molecular subgroup within the GBM $I D H$-wildtype.

\section{Abbreviations \\ ATRX: Alpha thalassemia/mental retardation syndrome X-linked; CRT: Chemo- radiotherapy; EGFR: Epidermal Growth Factor Receptor; FISH: Fluorescent In Situ Hybridization; GBM: Glioblastomas; HR: Hazard Ratio; IDH: Isocitrate dehydrogenase; IPOLFG: Unidade de Investigação em Patobiologia Molecular of Instituto Português de Oncologia de Lisboa Francisco Gentil; \\ ISCN: International System of Human Cytogenetic Nomenclature; MGMT: O- 6-methylguanine-DNA methyltransferase; MLPA: Multiplex Ligation- dependent Probe Amplification; NOS: Not Otherwise Specified; PCR: Polymerase Chain Reaction; PI3K-Akt: Phosphatidylinositol-3-kinase -protein kinase B; PTEN: Phosphatase and Tensin homologue; RT: Radiotherapy; TERT: Telomerase Reverse Transcriptase; TP53: Tumor Protein 53; WHO: World Health Organization}

\section{Acknowledgements}

The authors would like to thank Neurology Department of Instituto Português de Oncologia Lisboa Francisco Gentil and the financial support to Liga Portuguesa Contra o Cancro, Núcleo Regional Sul (LPCC-NRS), iNOVA4Health Research Unit (LISBOA-01-0145-FEDER-007344), which is cofunded by FCT/Ministério da Ciência e do Ensino Superior, through national funds, and by FEDER under the PT2020 Partnership Agreement.

\section{Authors' contributions}

All authors read and approved the final manuscript. All authors have made a sufficient contribution to the work. Concept and design: MP and LR. Experiments and procedures: CB, AZ, ARM, CM, IC, MM, JMBM, LR, Data analysis and writing the article: $C B, A Z, S E$ and MP.

\section{Funding}

This study was funded by Terry Fox grant 2018/2019. Marta Pojo was supported by Núcleo Regional Sul da Liga Portuguesa Contra o Cancro (NRSLPCC). The funding body was not involved in the design of the study and collection, analysis, and interpretation of data and in writing the manuscript.

\section{Availability of data and materials}

The datasets analyzed during the current study are available from the corresponding author on reasonable request.

\section{Ethics approval and consent to participate}

This study was approved by the IPOLFG Ethical Board Committee, (committee's reference number: UIC/1203). Study design and conduction of the study were performed accordingly to high standards of ethics. Written informed consent was obtained prior to any data acquisition, sample collection, or data analysis from the participants.

\section{Consent for publication}

Not applicable.

\section{Competing interests}

The authors declare that they have no competing interests.

\footnotetext{
Author details

'Unidade de Investigação em Patobiologia Molecular (UIPM) do Instituto Português de Oncologia de Lisboa Francisco Gentil E.P.E., Rua Prof. Lima Basto, 1099-023 Lisbon, Portugal. ${ }^{2}$ Serviço de Neurologia do Instituto Português de Oncologia de Lisboa Francisco Gentil E.P.E., Rua Prof. Lima Basto, 1099-023 Lisbon, Portugal. ${ }^{3}$ Faculty of Health Sciences, University of Beira Interior, 6200-506 Covilhã, Portugal. ${ }^{4}$ Unidade de Investigação Clínica (UIC) do Instituto Português de Oncologia de Lisboa Francisco Gentil E.P.E., Rua Prof. Lima Basto, 1099-023 Porto, Portugal. ${ }^{5}$ Serviço de Anatomia Patológica do Instituto Português de Oncologia de Lisboa Francisco Gentil E.P.E, Rua Prof. Lima Basto, 1099-023 Lisbon, Portugal.
} 
Received: 1 April 2019 Accepted: 20 September 2019

Published online: 17 October 2019

\section{References}

1. Ostrom QT, Bauchet L, Davis FG, Deltour I, Fisher JL, Langer CE, et al. The epidemiology of glioma in adults: a "state of the science" review. NeuroOncology. 2014;16(7):896-913.

2. Ferris SP, Hofmann JW, Solomon DA, Perry A. Characterization of gliomas: from morphology to molecules. Virchows Arch. 2017;471(2):257-69.

3. Reifenberger G, Wirsching HG, Knobbe-Thomsen CB, Weller M. Advances in the molecular genetics of gliomas-implications for classification and therapy. Nat Rev Clin Oncol. 2017;14(7):434-52.

4. Louis DN, Perry A, Reifenberger G, von Deimling A, Figarella-Branger D, Cavenee WK, et al. The 2016 World Health Organization classification of Tumors of the central nervous system: a summary. Acta Neuropathol. 2016; 131(6):803-20.

5. Foote MB, Papadopoulos N, Diaz LA. Genetic classification of Gliomas: refining histopathology. Cancer Cell. 2015;28(1):9-11.

6. Szopa W, Burley TA, Kramer-Marek G, Kaspera W. Diagnostic and therapeutic biomarkers in glioblastoma: current status and future perspectives. Biomed Res Int. 2017;2017(8013575):1-13.

7. Bollam SR, Berens ME, Dhruv HD. When the ends are really the beginnings: targeting telomerase for treatment of GBM. Curr Neurol Neurosci Rep. 2018;18(4):15.

8. Bryan TM, Cech TR. Telomerase and the maintenance of chromosome ends. Curr Opin Cell Biol. 1999;11(3):318-24.

9. Bell RJA, Rube HT, Xavier-Magalhães A, Costa BM, Mancini A, Song JS, et al. Understanding TERT promoter mutations: a common path to immortality. Mol Cancer Res. 2016;14(4):315-23.

10. Amorim JP, Santos G, Vinagre J, Soares P. The role of ATRX in the alternative lengthening of telomeres (ALT) phenotype. Genes. 2016;7(9):66.

11. Libermann TA, Nusbaum HR, Razon N, Kris R, Lax I, Soreq H, et al. Amplification, enhanced expression and possible rearrangement of EGF receptor gene in primary human brain tumours of glial origin. Nature. 1985; 313(5998):144-7.

12. Wong AJ, Bignert SH, Bignert DD, Kinzler KW, Hamilton SR, Vogelstein B. Increased expression of the epidermal growth factor receptor gene in malignant gliomas is invariably associated with gene amplification (oncogenes/in situ hybridization/chromosomes). Med Sci. 1987;84(19):6899-903.

13. Lai K, Killingsworth MC, Lee CS. Gene of the month: PIK3CA. J Clin Pathol. 2015;68(4):253-7.

14. Cancer Genome Atlas Research Network. Comprehensive genomic characterization defines human glioblastoma genes and core pathways. Nature. 2008;455(7216):1061-8.

15. Verhaak RGW, Hoadley KA, Purdom E, Wang V, Qi Y, Wilkerson MD, et al. Integrated genomic analysis identifies clinically relevant subtypes of Glioblastoma characterized by abnormalities in PDGFRA, IDH1, EGFR, and NF1. Cancer Cell. 2010;17(1):98-110.

16. Srividya MR, Thota B, Shailaja BC, Arivazhagan A, Thennarasu K, Chandramouli BA, et al. Homozygous 10q23/PTEN deletion and its impact on outcome in glioblastoma: a prospective translational study on a uniformly treated cohort of adult patients. Neuropathology. 2011;31(4):376-83.

17. Carico C, Nuño M, Mukherjee D, Elramsisy A, Dantis J, Hu J, et al. Loss of PTEN is not associated with poor survival in newly diagnosed glioblastoma patients of the temozolomide era. PLoS One. 2012;7(3):e33684.

18. Eckel-Passow JE, Lachance DH, Molinaro AM, Walsh KM, Decker PA, Sicotte $\mathrm{H}$, et al. Glioma groups based on 1p/19q, IDH, and TERT promoter mutations in Tumors. N Engl J Med. 2015;372(26):2499-508.

19. Lee $Y$, Koh J, Kim S-I, Won JK, Park C-K, Choi SH, et al. The frequency and prognostic effect of TERT promoter mutation in diffuse gliomas. Acta Neuropathol Commun. 2017;5(1):62.

20. Nguyen HN, Lie A, Li T, Chowdhury R, Liu F, Ozer B, et al. Human TERT promoter mutation enables survival advantage from MGMT promoter methylation in IDH1 wild-type primary glioblastoma treated by standard chemoradiotherapy. Neuro-Oncology. 2017;19(3):394-404.

21. Quan AL, Barnett GH, Lee SY, Vogelbaum MA, Toms SA, Staugaitis SM, et al. Epidermal growth factor receptor amplification does not have prognostic significance in patients with glioblastoma multiforme. Int J Radiat Oncol Biol Phys. 2005;63(3):695-703.

22. Shinojima N, Tada K, Shiraishi S, Kamiryo T, Kochi M, Nakamura H, et al. Prognostic value of epidermal growth factor receptor in patients with glioblastoma multiforme. Cancer Res. 2003;63(20):6962-70.
23. Chen JR, Xu HZ, Yao Y, Qin ZY. Prognostic value of epidermal growth factor receptor amplification and EGFRvIII in glioblastoma: meta-analysis. Acta Neurol Scand. 2015;132(5):310-22.

24. Hegi ME, Diserens A-C, Godard S, Dietrich P-Y, Regli L, Ostermann S, et al. Clinical trial substantiates the predictive value of 0-6-methylguanine-DNA methyltransferase promoter methylation in glioblastoma patients treated with temozolomide. Clin Cancer Res. 2004;10(6):1871-4.

25. Hegi ME, Diserens A-C, Gorlia T, Hamou M-F, de Tribolet N, Weller M, et al. MGMT gene silencing and benefit from temozolomide in glioblastoma. N Engl J Med. 2005;352(10):997-1003.

26. Chinot OL, Barrié M, Fuentes S, Eudes N, Lancelot S, Metellus P, et al. Correlation between O6-methylguanine-DNA methyltransferase and survival in inoperable newly diagnosed glioblastoma patients treated with neoadjuvant temozolomide. J Clin Oncol. 2007;25(12):1470-5.

27. Wick W, Meisner C, Hentschel B, Platten M, Schilling A, Wiestler B, et al. Prognostic or predictive value of MGMT promoter methylation in gliomas depends on IDH1 mutation. Neurology. 2013;81(17):1515-22.

28. Wick W, Weller M, van den Bent M, Sanson M, Weiler M, von Deimling A, et al. MGMT testing--the challenges for biomarker-based glioma treatment. Nat Rev Neurol. 2014;10(7):372-85.

29. van den Bent MJ, Weller M, Wen PY, Kros JM, Aldape K, Chang S. A clinical perspective on the 2016 WHO brain tumor classification and routine molecular diagnostics. Neuro-Oncology. 2017;19(5):614-24.

30. van den Bent MJ, Dubbink HJ, Sanson M, van der Lee-Haarloo CR, Hegi M, Jeuken JWM, et al. MGMT promoter methylation is prognostic but not predictive for outcome to adjuvant PCV chemotherapy in anaplastic oligodendroglial tumors: a report from EORTC brain tumor group study 26951. J Clin Oncol. 2009;27(35):5881-6.

31. McGowan-Jordan J, Simons A, Schmid J. ISCN - An International System for Human Cytogenomic Nomenclature. Basel; New York: Karger; 2016. p. 139.

32. Iuchi T, Sugiyama T, Ohira M, Kageyama H, Yokoi S, Sakaida T, et al. Clinical significance of the 2016 WHO classification in Japanese patients with gliomas. Brain Tumor Pathol. 2018;35(2):71-80.

33. Tabouret E, Nguyen AT, Dehais C, Carpentier C, Ducray F, Idbaih A, et al. Prognostic impact of the 2016 WHO classification of diffuse gliomas in the French POLA cohort. Acta Neuropathol. 2016;132(4):625-34.

34. Parsons DW, Jones S, Zhang X, Lin JC, Leary RJ, Angenendt P, et al. An integrated genomic analysis of human glioblastoma multiforme. Science. 2008;321(5897):1807-12.

35. Griffin CA, Burger P, Morsberger L, Yonescu R, Swierczynski S, Weingart JD, et al. Identification of $\operatorname{der}(1 ; 19)(q 10 ; p 10)$ in five oligodendrogliomas suggests mechanism of concurrent $1 p$ and $19 q$ loss. J Neuropathol Exp Neurol. 2006;65(10):988-94.

36. Jenkins RB, Blair H, Ballman KV, Giannini C, Arusell RM, Law M, et al. A t(1; 19)(q10;p10) mediates the combined deletions of $1 p$ and $19 q$ and predicts a better prognosis of patients with oligodendroglioma. Cancer Res. 2006; 66(20):9852-61.

37. Wang P, Wu J, Ma S, Zhang L, Yao J, Hoadley KA, et al. Oncometabolite D-2Hydroxyglutarate inhibits ALKBH DNA repair enzymes and sensitizes IDH mutant cells to alkylating agents. Cell Rep. 2015;13(11):2353-61.

38. Sturm D, Witt H, Hovestadt V, Khuong-Quang DA, Jones DTW Konermann C, et al. Hotspot mutations in H3F3A and IDH1 define distinct epigenetic and biological subgroups of Glioblastoma. Cancer Cell. 2012;22(4):425-37.

39. Waitkus MS, Diplas BH, Yan H. Isocitrate dehydrogenase mutations in gliomas. Neuro-Oncology. 2016;18(1):16-26.

40. Sartori E, Langer R, Vassella E, Hewer E, Schucht P, Zlobec I, et al. Low coexpression of epidermal growth factor receptor and its chaperone heat shock protein 90 is associated with worse prognosis in primary glioblastoma. IDH-wild-Type Oncol Rep. 2017;38(4):2394-400.

41. Simmons ML, Lamborn KR, Takahashi M, Chen P, Israel MA, Berger MS, et al. Analysis of complex relationships between age, p53, epidermal growth factor receptor, and survival in glioblastoma patients. Cancer Res. 2001;61(3):1122-8.

42. Sarkaria JN, Carlson BL, Schroeder MA, Grogan P, Brown PD, Giannini C, et al. Use of an orthotopic xenograft model for assessing the effect of epidermal growth factor receptor amplification on glioblastoma radiation response. Clin Cancer Res. 2006;12(7 I):2264-71.

43. Das AK, Sato M, Story MD, Peyton M, Graves R, Redpath S, et al. Nonsmall cell lung cancers with kinase domain mutations in the epidermal growth factor receptor are sensitive to ionizing radiation. Cancer Res. 2006;66(19):9601-8. 
44. Xie B, Sun L, Cheng Y, Zhou J, Zheng J, Zhang W. Epidermal growth factor receptor gene mutations in non-small-cell lung cancer cells are associated with increased radiosensitivity in vitro. Cancer Manag Res. 2018;10:3551-60.

45. Gupta AK, Soto DE, Feldman MD, Goldsmith JD, Mick R, Hahn SM, et al. Signaling pathways in NSCLC as a predictor of outcome and response to therapy. Lung. 2004;182(3):151-62.

46. McEllin B, Camacho CV, Mukherjee B, Hahm B, Tomimatsu N, Bachoo RM, et al. PTEN loss compromises homologous recombination repair in astrocytes: implications for glioblastoma therapy with temozolomide or poly(ADP-ribose) polymerase inhibitors. Cancer Res. 2010;70(13):5457-64

47. Slipicevic A, Holm R, Nguyen MTP, Bøhler PJ, Davidson B, Flørenes VA. Expression of activated Akt and PTEN in malignant melanomas: relationship with clinical outcome. Am J Clin Pathol. 2005;124(4):528-36.

48. Panigrahi AR, Pinder SE, Chan SY, Paish EC, Robertson JFR, Ellis IO. The role of PTEN and its signalling pathways, including AKT, in breast cancer; an assessment of relationships with other prognostic factors and with outcome. J Pathol. 2004;204(1):93-100.

49. Ma J, Benitez JA, Li J, Miki S, Ponte de Albuquerque C, Galatro T, et al. Inhibition of nuclear PTEN tyrosine phosphorylation enhances Glioma radiation sensitivity through attenuated DNA repair. Cancer Cell. 2019;35(3):504-18.

50. Xu W, Yang H, Liu Y, Yang Y, Wang P, Kim SH, et al. Oncometabolite 2hydroxyglutarate is a competitive inhibitor of a-ketoglutarate-dependent dioxygenases. Cancer Cell. 2011;19(1):17-30.

51. Rivera AL, Pelloski CE, Gilbert MR, Colman H, De La Cruz C, Sulman EP, et al. MGMT promoter methylation is predictive of response to radiotherapy and prognostic in the absence of adjuvant alkylating chemotherapy for glioblastoma. Neuro-Oncology. 2010;12(2):116-21.

\section{Publisher's Note}

Springer Nature remains neutral with regard to jurisdictional claims in published maps and institutional affiliations.

Ready to submit your research? Choose BMC and benefit from:

- fast, convenient online submission

- thorough peer review by experienced researchers in your field

- rapid publication on acceptance

- support for research data, including large and complex data types

- gold Open Access which fosters wider collaboration and increased citations

- maximum visibility for your research: over $100 \mathrm{M}$ website views per year

At $\mathrm{BMC}$, research is always in progress.

Learn more biomedcentral.com/submissions 